\title{
Utility of endobronchial ultrasound-guided mediastinal lymph node biopsy in patients with non-small cell lung cancer
}

\author{
Benjamin E. Lee, MD, ${ }^{\mathrm{a}, \mathrm{b}}$ Elaine Kletsman, PA, ${ }^{\mathrm{a}, \mathrm{b}}$ John R. Rutledge, MAS, ${ }^{\mathrm{a}}$ and Robert J. Korst, $\mathrm{MD}^{\mathrm{a}, \mathrm{b}}$
}

Objective: Invasive mediastinal biopsy is often necessary in the evaluation of non-small cell lung cancer (NSCLC), and mediastinoscopy has long been considered the reference standard. However, the emergence of endobronchial ultrasound-guided transbronchial needle aspiration (EBUS-TBNA) has resulted in controversy regarding it represents a suitable replacement for mediastinoscopy. We chose to determine the utility of EBUS-TBNA in evaluating the mediastinum in patients with NSCLC.

Methods: The present study was a retrospective review of a prospective database of consecutive patients with NSCLC who underwent EBUS-TBNA for mediastinal evaluation from 2009 to 2011. The sensitivity, specificity, negative predictive value, and accuracy of EBUS-TBNA are reported. Also reported are the size of the lymph nodes biopsied and the number of instances in which EBUS-TBNA obviated the need for cervical mediastinoscopy.

Results: A total of 73 patients had a total of 140 mediastinal stations biopsied using EBUS-TBNA. Of the 73 patients, 30 had benign findings and underwent surgical resection, 1 of whom was found to have stage N2 disease. Of the remaining patients, 42 had a positive result and 1 had nondiagnostic biopsy findings for which malignancy was confirmed by mediastinoscopy. Mediastinoscopy would have changed the tumor stage and treatment planning in only $2(2.7 \%)$ of the 73 patients. Overall, EBUS-TBNA had a sensitivity of $95 \%$, a specificity of $100 \%$, a negative predictive value of $94 \%$, and an accuracy of $97 \%$.

Conclusions: EBUS-TBNA might be a feasible option for most patients with NSCLC for whom histologic assessment of the mediastinum is necessary. The rates of nondiagnostic and false-negative biopsy findings using EBUS-TBNA were low, small subcentimeter nodes could be routinely biopsied, and most patients with a radiographically positive mediastinum had their disease pathologically confirmed. (J Thorac Cardiovasc Surg 2012;143:585-90)

Non-small cell lung cancer (NSCLC) frequently metastasizes to the mediastinal lymph nodes, representing a process that affects the tumors stage and treatment strategy. Cervical mediastinoscopy with biopsies has been the reference standard for staging the mediastinum of patients with NSCLC for decades. ${ }^{1}$ However, the use of endobronchial ultrasound-guided transbronchial needle aspiration (EBUSTBNA) is gaining popularity among physicians involved in the staging of lung cancer. As a result of this new technology, a debate has arisen regarding the accuracy of EBUS-TBNA in correctly determining the presence of mediastinal nodal disease and the effect of EBUS-TBNA on the need to perform cervical mediastinoscopy in this patient population.

\footnotetext{
From the Daniel and Gloria Blumenthal Cancer Center, ${ }^{\mathrm{a}}$ Paramus, NJ; and Division of Thoracic Surgery, ${ }^{\mathrm{b}}$ Department of Surgery, Valley Hospital/Valley Health System, Ridgewood, NJ.

Disclosures: Authors have nothing to disclose with regard to commercial support.

Read at the 37th Annual Meeting of The Western Thoracic Surgery Association, Colorado Springs, Colorado, June 22-25, 2011.

Received for publication June 20, 2011; revisions received Oct 28, 2011; accepted for publication Nov 9, 2011; available ahead of print Dec 12, 2011.

Address for reprints: Benjamin E. Lee, MD, Division of Thoracic Surgery, Department of Surgery, Valley Hospital/Valley Health System, 1 Valley Health Plaza, Paramus, NJ 07652 (E-mail: leebe@ valleyhealth.com).

$0022-5223 / \$ 36.00$

Copyright (C) 2012 by The American Association for Thoracic Surgery

doi:10.1016/j.jtcvs.2011.11.022
}

Some investigators have found EBUS-TBNA to provide an accurate examination of the mediastinum. However, others have maintained that cervical mediastinoscopy should remain the definitive biopsy procedure, citing a high falsenegative rate associated with EBUS-guided biopsies. ${ }^{2-5}$

Given this controversy, we sought to determine the role of EBUS-TBNA in evaluating the mediastinum in our patients with NSCLC. Specifically, the purpose of the present study was to determine the (1) efficacy of EBUS-TBNA, (2) how often EBUS-TBNA yields a positive result in patients with a radiographically positive mediastinum, and (3) the nondiagnostic biopsy rate.

\section{METHODS}

\section{Study Design and Patient Cohort}

The present study was a retrospective review of a prospectively maintained database of patients undergoing EBUS-TBNA. The institutional review board waived the need for informed consent. Since September 2009 all patients who met the standard criteria for cervical mediastinoscopy because of proven or suspected lung cancer instead underwent EBUS-TBNA Generally, this group of patients included those requiring mediastinal staging before definitive resection (stage IB to IIIA) and those with radiographic stage IIIB disease (large and/or confluent contralateral noda mass) or stage IV disease (brain metastasis) who required a tissue diagnosis. 


$$
\begin{aligned}
& \text { Abbreviations and Acronyms } \\
& \begin{aligned}
\text { CT } & =\text { computed tomography } \\
\text { EBUS- } & =\text { endobronchial ultrasound-guided } \\
\text { TBNA } & \text { transbronchial needle aspiration } \\
\text { L } & =\text { level } \\
\text { LL } & =\text { left level } \\
\text { NSCLC } & =\text { non-small cell lung cancer } \\
\text { PET } & =\text { positron emission tomography } \\
\text { RL } & =\text { right level }
\end{aligned}
\end{aligned}
$$

The patients were classified according to whether the mediastinum was radiographically positive, defined as positive findings on computed tomography (CT) and/or positron emission tomography (PET). Positive CT findings meant that lymph nodes in right level (RL) 2, RL4, level 7 (L7), and/or left level (LL) 4 stations had a short axis diameter of greater than $1 \mathrm{~cm}$. The PET findings were interpreted by institutional radiologists as positive or negative, comparing the lymph node to the uptake in the mediastinal blood pool. If positive, the maximum standardized uptake value was reported.

Patients with radiographically positive mediastinal lymph nodes outside the paratracheal and subcarinal areas (L5, L6, L8, L9) were excluded from the present study because these regions are outside the reach of cervical mediastinoscopy. These patients underwent staging using other modalities, including thoracoscopy and/or endoesophageal ultrasonography.

\section{EBUS-Guided Mediastinal Biopsies}

All EBUS-TBNA procedures were performed with the patient under general anesthesia through a laryngeal mask airway using an EBUSTBNA bronchoscope (Olympus Exera BF-UC180F; Olympus Imaging America, Center Valley, Pa). Lymph node biopsies were performed using either a 21- or 22-gauge EBUS-TBNA biopsy needle, from highest to lowest stage (eg, N3 to N2 to N1). The lymph node size, in and of itself, was not a determinate of whether biopsy was attempted. Efforts were made to biopsy all nodal basins in all patients undergoing preoperative staging, unless no obvious nodal tissue was detected in a given station using EBUS. Patients with intraoperatively confirmed stage IIIB disease did not undergo multistation biopsies, because therapy would not be affected by the acquisition of more tissue.

Once the biopsy needle was introduced into a candidate lymph node and suction applied, multiple needle passes $(>25)$ were made, with manipulation of the bronchoscope to ensure thorough nodal sampling. The specimens were placed in cytolyte, and, although rapid on-site evaluation of slides was available, it was not routinely performed. The decision regarding repeat biopsy of the same lymph node was determined by gross inspection of the biopsy specimens in the cytolyte. A biopsy was considered diagnostic if it either contained tumor ("positive" EBUS) or benign lymph node tissue ("negative" EBUS; lymphocytes and/or histiocytes). Complications from the EBUS-guided biopsy procedure were recorded.

\section{Confirmation of EBUS-TBNA Results}

The EBUS-TBNA results were confirmed in selected patients by the additional acquisition of tissue:

Patients with negative preoperative staging EBUS-TBNA findings underwent surgical resection using either a thoracoscopic (video-assisted thoracic surgery) or an open thoracotomy approach. During all right-sided resections, RL4 and L7, L10, and L11 were dissected; on the left side, L5, L6, L7, L10, and L11 were dissected.
Patients with a radiographically positive mediastinum and nondiagnostic EBUS-TBNA findings underwent cervical mediastinoscopy, in which attempts were made to biopsy RL2, RL4, LL4, and L7 nodal stations.

Patients with a radiographically positive mediastinum and negative EBUS-TBNA findings underwent either resection (as above) or cervical mediastinoscopy (as above), followed by surgical resection, if negative. The decision to go directly to resection in these cases was on a case-by-case basis.

Patients with positive EBUS-TBNA findings, with stage IIIA and an adequate cardiopulmonary reserve underwent preoperative chemotherapy followed by surgical resection using an open thoracotomy approach (as above), when appropriate.

Of note, the patients with positive mediastinal biopsy findings who were not surgical candidates because of an advanced disease stage (IIIB) or unresectable disease did not undergo additional tissue acquisition from the mediastinum.

\section{Clinical Stage}

The clinical stage was derived from the findings from all noninvasive and invasive procedures performed before surgical resection, when appropriate. The 7th edition of the American Joint Committee on Cancer TNM staging system for NSCLC was used.

\section{Statistical Analysis}

The median and interquartile range were determined for lymph node size and maximum standard uptake value as determined from the $\mathrm{CT}$ and PET scans, respectively. These quantities were tabulated according to the result of the EBUS-TBNA procedure. The sensitivity, specificity, negative predictive value, and accuracy of EBUS-TBNA were calculated according to the biopsy results of the lymph nodes that had undergone both EBUS-TBNA and surgical confirmation. The $95 \%$ confidence intervals for these values are presented. ${ }^{6}$ Sensitivity was calculated as the number of true-positive results/(number of true-positive results + number of false-negative results). Specificity was calculated as the number of truenegative results/(number of false-positive results + number of truenegative results). The negative predictive value was calculated as the number of true-negative results/(number of false-negative results + number of true-negative results). Accuracy was calculated as the number of EBUS-TBNA results confirmed by surgical resection/total number of EBUS-TBNA procedures performed. It was assumed that any lymph node deemed malignant by EBUS-TBNA was truly malignant.

\section{RESULTS \\ Patient Characteristics}

From September 2009 to April 2011, 73 patients met the criteria for inclusion in the present study. The clinical characteristics of the study cohort are listed in Table 1. Of the 73 patients, $57(78 \%)$ had a radiographically positive mediastinum using either the CT criteria alone $(\mathrm{n}=26)$, PET criteria alone $(n=1)$, or combined positive PET and CT findings $(\mathrm{n}=30)$. Twelve patients had undergone no PET before EBUS-TBNA. The median maximum standardized uptake value in the patients with positive PET findings was 8.8 (interquartile range, 5.28-13), and the median nodal size (short axis) of the patients with positive CT findings was $2 \mathrm{~cm}$ (interquartile range, 1.5-2.6). In the $30 \mathrm{pa}-$ tients with presumed early-stage disease (stage 1-2), 77 lymph node stations were sampled (2.6 stations/patient). 
TABLE 1. Clinical features $(n=73)$

\begin{tabular}{|c|c|}
\hline Characteristic & Patients (n) \\
\hline \multicolumn{2}{|l|}{ Gender } \\
\hline Male & $34(47)$ \\
\hline Female & $39(53)$ \\
\hline \multicolumn{2}{|l|}{ Age (y) } \\
\hline Mean & 69.2 \\
\hline Range & $36-91$ \\
\hline \multicolumn{2}{|c|}{ Primary tumor location } \\
\hline RUL & $20(27)$ \\
\hline RML & $2(3)$ \\
\hline RLL & $15(21)$ \\
\hline LUL & $19(26)$ \\
\hline LLL & $12(16)$ \\
\hline None & $5(7)$ \\
\hline \multicolumn{2}{|l|}{ Clinical stage } \\
\hline IA & $10(14)$ \\
\hline IB & $10(14)$ \\
\hline IIA & $3(4)$ \\
\hline IIB & $3(4)$ \\
\hline IIIA & $20(27)$ \\
\hline $\mathrm{T} 1-\mathrm{T} 3 \mathrm{~N} 2$ & 17 \\
\hline $\mathrm{T} 3 \mathrm{~N} 1$ & 2 \\
\hline T4N0 & 1 \\
\hline IIIB & $12(16)$ \\
\hline $\mathrm{T} 1-\mathrm{T} 3 \mathrm{~N} 3$ & 10 \\
\hline $\mathrm{T} 4 \mathrm{~N} 2$ & 2 \\
\hline IVA & $3(4)$ \\
\hline IVB & $12(16)$ \\
\hline \multicolumn{2}{|l|}{ Histologic finding } \\
\hline Adenocarcinoma & $48(66)$ \\
\hline Squamous & $18(25)$ \\
\hline Large cell & $3(4)$ \\
\hline Carcinosarcoma & $2(3)$ \\
\hline Adenosquamous & $1(1.4)$ \\
\hline Carcinoid & $1(1.4)$ \\
\hline
\end{tabular}

\section{Clinical Outcome and Replacement of Cervical Mediastinoscopy With EBUS-TBNA}

A flow diagram demonstrating the clinical outcome of all 73 patients in the present study, according to the radiographic status of the mediastinum, is shown in Figure 1. Significantly, $42(74 \%)$ of the 57 patients with a radiographically positive mediastinum had malignant disease in the mediastinal lymph nodes proven using EBUSTBNA. In the entire cohort, only 4 patients underwent cervical mediastinoscopy after EBUS-TBNA. In 3 of these patients, the EBUS-TBNA findings were negative despite a radiographically positive mediastinum. In all 3 , the mediastinal lymph nodes were negative after both cervical mediastinoscopy and subsequent video-assisted thoracic surgery lobectomy. The fourth patient underwent cervical mediastinoscopy after non-diagnostic EBUS-TBNA for

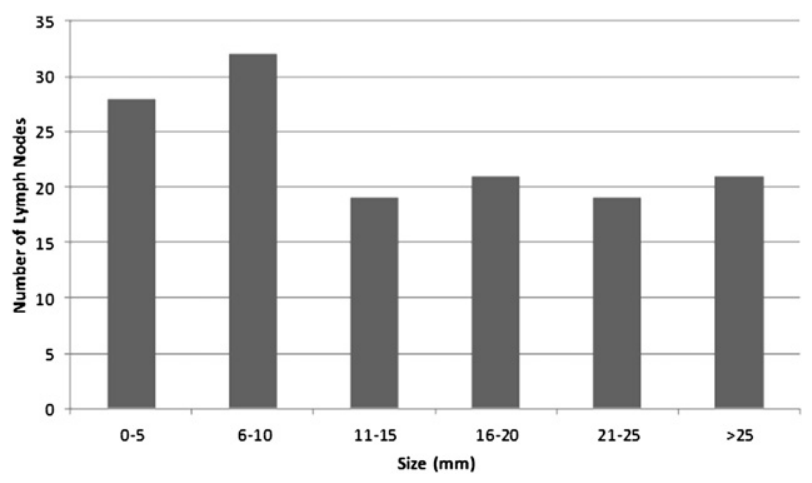

FIGURE 1. Distribution of lymph node sizes biopsied using endobronchial ultrasound-guided transbronchial needle aspiration.

a radiographically positive mediastinum, yielding a positive result (RL4).

Overall, cervical mediastinoscopy was not performed in $69(95 \%)$ of the 73 patients, who, before EBUS-TBNA, would have undergone the more invasive procedure. Furthermore, only 1 patient had positive mediastinoscopy findings in the present study, with a single additional patient undergoing resection with $\mathrm{N} 2$ disease discovered, despite negative EBUS-TBNA findings (no preresection mediastinoscopy performed). Therefore, cervical mediastinoscopy would have changed the tumor stage and treatment planning in only $2(2.7 \%)$ of 73 patients. The overall sensitivity, specificity, positive predictive value, negative predictive value, and accuracy for EBUS-TBNA was $95 \%$ (42/44), 100\% (29/29), 100\% (42/42), 94\% (29/31), and 97\% (71/73), respectively. Only 1 complication (atrial fibrillation) occurred after EBUS-TBNA $(1.4 \%)$.

\section{Ability of EBUS-TBNA to Detect Mediastinal Nodal Disease}

In the 73 patients, 140 lymph node stations were biopsied using EBUS-TBNA. The distribution of lymph node sizes biopsied using EBUS-TBNA is shown in Figure 2. Of the EBUS-TBNA biopsies were positive in 51 and negative in 88 , with a single nondiagnostic biopsy (RL4). No lymph nodes were biopsied in RL2. The EBUS-guided biopsy results, radiographic characteristics, sensitivity, specificity, negative predictive value, and accuracy according to nodal station are listed in Table 2. Only positive biopsy and negative biopsy findings confirmed by either mediastinoscopy or at surgical resection were included in the present analysis (100/140).

\section{DISCUSSION}

The utility of EBUS-TBNA in staging the mediastinum in patients with NSCLC remains controversial (Table 3). ${ }^{4,7-10}$ Recent data and published reviews have suggested that many radiographically suspicious nodal stations are either 


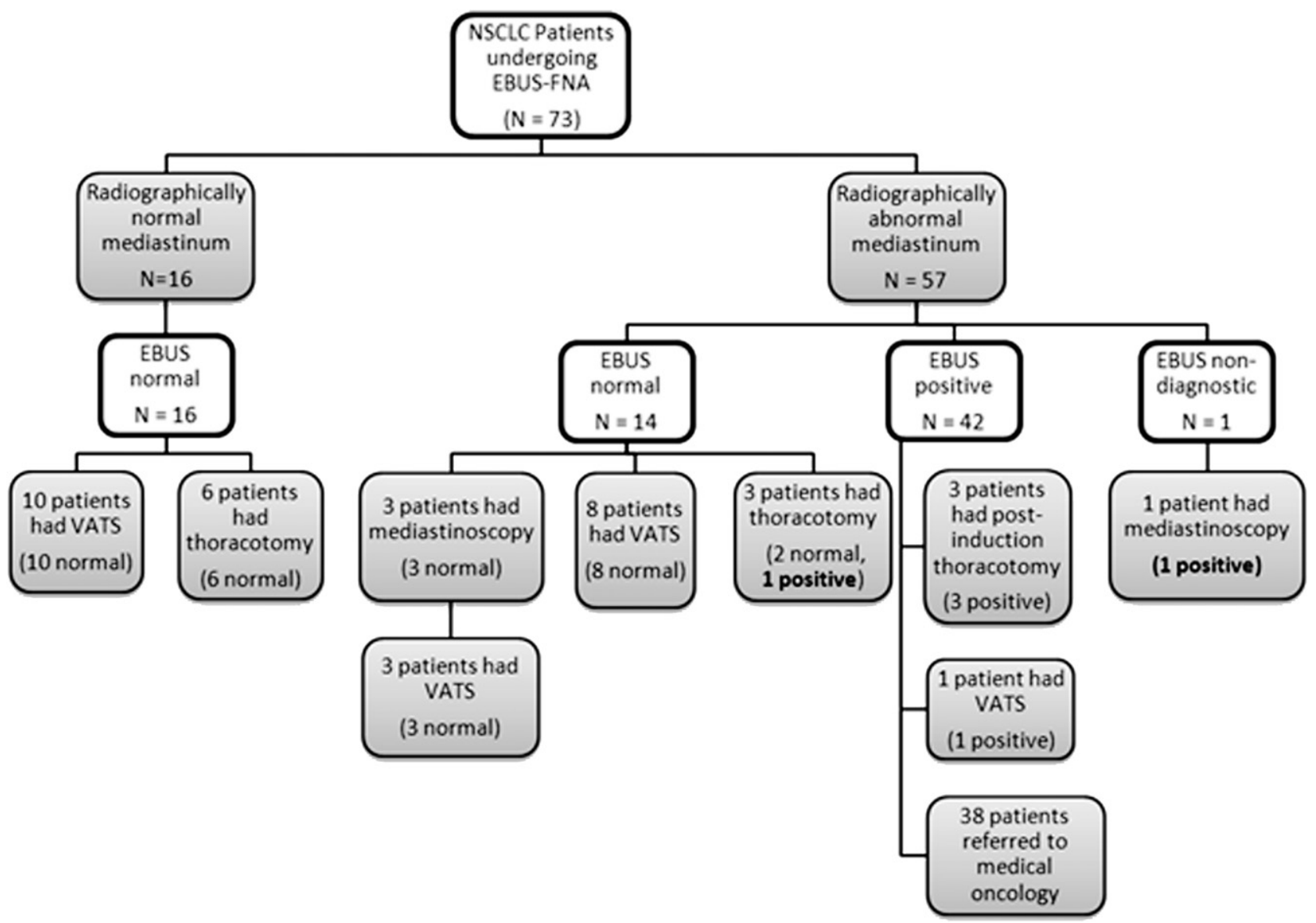

FIGURE 2. Endobronchial ultrasound-guided transbronchial needle aspiration (EBUS-TBNA) and patient outcomes. VATS, Video-assisted thoracic surgery.

not biopsied during EBUS-TNBA or the biopsy results are nondiagnostic. ${ }^{3-5}$ In addition, many experts have recommended that, because of the significant false-negative results obtained with EBUS-TBNA, negative EBUS-TBNA findings need to be confirmed by cervical mediastinoscopy. ${ }^{1-5,11}$ The data from the present study contradicts some of these concerns regarding EBUS-TBNA. The data suggest that EBUS-TBNA is safe and accurate in detecting

TABLE 2. Characteristics of EBUS-guided lymph node biopsies stratified by nodal station $(n=100)$

\begin{tabular}{|c|c|c|c|c|c|c|c|c|}
\hline $\begin{array}{c}\begin{array}{c}\text { Station } \\
\text { (node no.) }\end{array} \\
\end{array}$ & $\begin{array}{c}\text { EBUS-FNA } \\
\text { result (n) }\end{array}$ & CT size* (mm) & $\begin{array}{c}\text { MaxSUV } \\
\text { Median (IQR) }\end{array}$ & TP, FN, FP, TN & Sensitivity $\dagger$ & Specificity $\dagger$ & $\mathbf{N P V} \dagger$ & Accuracy $\dagger$ \\
\hline 4R (41) & & & & $24,1,0,16$ & $96(78-100)$ & $100(76-100)$ & $94(69-100)$ & $98(86-100)$ \\
\hline Positive & 24 & $24(17-37.6)$ & $8(4.5-13)$ & & & & & \\
\hline Negative & 16 & $7(5-15)$ & $0(0-0)$ & & & & & \\
\hline ND & 1 & 16 (NA) & 5.2 (NA) & & & & & \\
\hline $4 \mathrm{~L}(6)$ & & & & $4,0,0,2$ & $100(40-100)$ & $100(20-100)$ & $100(20-100)$ & $100(52-100)$ \\
\hline Positive & 4 & 36.6 (NA) & 12 (NA) & & & & & \\
\hline Negative & 2 & 5 (NA) & 0 (NA) & & & & & \\
\hline ND & 0 & 0 & 0 & & & & & \\
\hline $7(53)$ & & & & $23,1,0,29$ & $96(77-100)$ & $100(85-100)$ & $97(81-100)$ & $98(89-100)$ \\
\hline Positive & 23 & $21.5(16.5-28.3)$ & $8.9(5.2-12.1)$ & & & & & \\
\hline Negative & 30 & $9(7-12.6)$ & $0(0-0)$ & & & & & \\
\hline ND & 0 & 0 & 0 & & & & & \\
\hline
\end{tabular}

Only EBUS-TBNA biopsies subsequently confirmed by either mediastinoscopy or surgical resection included. EBUS-TBNA, Endobronchial ultrasound-guided transbronchial needle aspiration; $C T$, computed tomography; $\operatorname{Max} S U V$, maximum standardized uptake value; $T P$, true-positive result; $F N$, false-negative result; $F P$, false-positive result; $T N$, truenegative result; $N P V$, negative predictive value; $N D$, nondiagnostic $N A$, not able to be calculated. *Data presented as median, with interquartile range in parentheses. $\dagger$ Data in parentheses are $95 \%$ confidence intervals. 
TABLE 3. Recent publications of EBUS-TBNA for NSCLC

\begin{tabular}{lccccc}
\hline \multicolumn{1}{c}{ Investigator } & Year & $\begin{array}{c}\text { Patients } \\
(\mathbf{n})\end{array}$ & $\begin{array}{c}\text { Sensitivity } \\
(\%)\end{array}$ & $\begin{array}{c}\text { NPV } \\
(\%)\end{array}$ & $\begin{array}{c}\text { Accuracy } \\
(\%)\end{array}$ \\
\hline Cerfolio et al $^{4}$ & 2010 & 72 & 57 & 79 & 83 \\
Yasufuku et al $^{7}$ & 2011 & 153 & 84.3 & 92.7 & 94.8 \\
Andrade et al $^{8}$ & 2010 & 98 & 87.9 & 84.4 & 91.7 \\
Hwango et al $^{9}$ & 2010 & 150 & 84.4 & 93.3 & 95.1 \\
Szlubowki et al $^{10}$ & 2009 & 226 & 89 & 83.5 & 92.9 \\
\hline
\end{tabular}

EBUS-TBNA, Endobronchial ultrasound-guided transbronchial needle aspiration; $N S C L C$, non-small cell lung cancer; $N P V$, negative predictive value.

mediastinal nodal disease, with both a low nondiagnostic biopsy rate and a low false-negative rate. In addition, diagnostic tissue can be successfully obtained from small (subcentimeter) lymph nodes. Finally, the performance of EBUS-guided mediastinal node biopsies might obviate the need for cervical mediastinoscopy in most patients with NSCLC requiring mediastinal assessment.

\section{Maximizing Effect of EBUS-TBNA in Mediastinum}

The results from the present study have demonstrated that EBUS-TBNA is effective in evaluating the mediastinum and that negative findings might not have to be followed by cervical mediastinoscopy. In particular, all the patients with a radiographically negative mediastinum and negative EBUS-TBNA findings were node negative on final pathologic staging. Furthermore, only 1 of 14 patients with a radiographically positive mediastinum and negative EBUS-TBNA findings had disease in the mediastinum on resection. Although this conclusion is in direct contrast to those from other published reports, several recent studies, as well as a recently reported prospective clinical trial that mandated routine cervical mediastinoscopy after every EBUS-TBNA, ${ }^{7-9}$ support our conclusion.

We propose that several factors might have played a role in optimizing the performance of EBUS-guided biopsies in the present experience. First, general anesthesia was used in all patients. It is conceivable that the absence of spontaneous respiration might have been beneficial, especially when performing biopsies of small (subcentimeter) lymph nodes. Second, a larger bore (21-gauge) biopsy needle was used for most biopsies, regardless of nodal size, which theoretically could have increased the volume of specimen acquired. Third, all EBUS-TBNA procedures were performed by the thoracic surgeons who ultimately diagnosed, staged, and resected all patients. This continuity of care for these complicated patients might have allowed for a more thorough staging evaluation with EBUS-TBNA. As an example, perhaps the persistence of the EBUS-TBNA operator to obtain adequate biopsies was affected by this continuity of care, especially when managing small nodes in difficult locations. Clearly, these subtle differences in technique need to be investigated further and directly compared with their counterparts (ie, sedation vs general anesthesia, 21- vs 22-gauge needle) to determine whether a true benefit exists.

\section{Ability of EBUS-TBNA to Replace Cervical Mediastinoscopy}

Because the experience from the present study did not directly compare EBUS-TBNA and cervical mediastinoscopy, we cannot conclude that EBUS-TBNA represents a suitable replacement for mediastinoscopy. However, in comparisons such as this, it is important to realize that cervical mediastinoscopy is also associated with a falsenegative rate and is therefore not truly a reference standard. Yasufuku and colleagues, ${ }^{7}$ in a rigorously conducted prospective trial, found that cervical mediastinoscopy "missed" nearly as many malignant nodes as did EBUSTBNA. They concluded that EBUS-TBNA might be able to replace cervical mediastinoscopy. ${ }^{7}$

The accuracy of EBUS-TBNA in evaluating the mediastinum of patients with NSCLC will probably never be identical in every operator's hands. Just as has been shown with other ultrasound-based technologies, the accuracy with EBUS-TBNA is most likely extremely user dependent. ${ }^{12}$ Inherent operator factors, such as the knowledge of mediastinal anatomy and spatial relationships and the aggressiveness in performing biopsies, play a role in every procedure and are difficult to control from institution to institution and from operator to operator. Perhaps each operator/institution will need to assess their strengths and weaknesses with regard to EBUS-TBNA and cervical mediastinoscopy and only then derive their own staging algorithm.

\section{Strengths and Limitations}

The strengths of the present analysis included the prospective nature of the data collection and the inclusion of only those nodal stations accessible using cervical mediastinoscopy. Thus, we chose not to complicate the study by including the evaluation of L5-L6 nodes because we believe these levels are not truly accessible using either EBUSTBNA or cervical mediastinoscopy. Another strength was the uniformity of the operators and infrastructure in the EBUS-TBNA program, with all procedures performed using the same techniques by only 2 operators.

The main limitation of the present study was that most EBUS-TBNA procedures were not followed by routine mediastinoscopy. For example, patients with positive biopsy results by EBUS-TBNA were assumed to have true disease and did not undergo confirmatory mediastinoscopy. Accordingly this might introduce the possibility of workup bias in the present study. Obviously, the most relevant method to determine whether EBUS-TBNA could replace mediastinoscopy would be a study in which every patient underwent both EBUS-TBNA and mediastinoscopy. However, such a strategy would require a formal 
clinical trial and informed consent, with the patients understanding that in many circumstances they would undergo an "unnecessary" procedure. Given our experience, combined with the recent published results of a clinical trial that considered this very question, such a trial would likely need to be performed as a multiinstitutional study.

Another limitation inherent in any study assessing mediastinal biopsy techniques is that all the mediastinal nodes will not be thoroughly dissected in every patient. Even with open thoracotomy and radical node dissection, specific nodal basins will not be dissected (eg, the paratracheal nodes during left-sided pulmonary resection). This is a limitation that is difficult to overcome given the standards of clinical care for patients with lung cancer.

The relatively small sample size was another limitation of the present study. Ideally, a multi-institutional randomized study focusing on mediastinal staging in patients with operable NSCLC would provide the best evidence in determining whether EBUS-TBNA is a suitable replacement for cervical mediastinoscopy.

In conclusion, our experience with EBUS-TBNA of the mediastinal lymph nodes for the evaluation of the mediastinum in patients with NSCLC suggests that this new technology is safe and accurate. The rates of nondiagnostic and false-negative biopsies were low, small subcentimeter nodes could be routinely biopsied, and most patients with a radiographically positive mediastinum had their disease pathologically confirmed by EBUS-TBNA.

\section{References}

1. Shrager JB. Mediastinoscopy: still the gold standard. Ann Thorac Surg. 2010;89: S2084-9.

2. Annema JT, van Meerbeeck JP, Rintoul RC, Dooms C, Deschepper E, Dekkers OM, et al. Mediastinoscopy vs. endosonography for mediastinal nodal staging of lung cancer. JAMA. 2010;304:2245-52.

3. Defranchi SA, Edell ES, Daniels CE, Prakash UB, Swanson KL, Utz JP, et al. Mediastinoscopy in patients with lung cancer and negative endobronchial ultrasound guided needle aspiration. Ann Thorac Surg. 2010;90:1753-8.

4. Cerfolio RJ, Bryant AS, Eloubeidi MA, Frederick PA, Minnich DJ, Harbour KC, et al. The true false negative rates of esophageal and endobronchial ultrasound in the staging of mediastinal lymph nodes in patients with non-small cell lung cancer. Ann Thorac Surg. 2010;90:427-34.

5. Detterbeck FC, Jantz MA, Wallace M, Vansteenkiste J, Silvestri GA. Invasive mediastinal staging of lung cancer: ACCP evidence-based clinical practice guidelines (2nd edition). Chest. 2007;132:202S-20S.

6. Newcombe RG. Two-sided confidence intervals for the single proportion: comparison of seven methods. Stat Med. 1998;17:857-72.

7. Yasufuku K, Pierre A, Darling GE, Perrot M, Waddell TK, Johnston MR, et al. A prospective controlled trial of endobronchial ultrasound-guided transbronchial needle aspiration compared to mediastinoscopy for mediastinal lymph node staging of lung cancer. Abstract presented at 2011 American Association for Thoracic Surgery.

8. Andrade RS, Groth SS, Rueth NM, D'Cunha J, Pambuccian SE, Maddaus MA. Evaluation of mediastinal lymph nodes with endobronchial ultrasound: the thoracic surgeon's perspective. J Thorac Cardiovasc Surg. 2010;139:578-83.

9. Hwango B, Lee GK, Lee HS, Lim KY, Lee SH, Kim HY, et al. Transbronchial and transesophageal fine-needle aspiration using an ultrasound bronchoscope in mediastinal staging of potentially operable lung cancer. Chest. 2010;138:795-802.

10. Szlubowki A, Kuzdza J, Kolodziej M, Soja J, Pankowski J, Obrochta A, et al. Endobronchial ultrasound-guided needle aspiration in the non-small cell lung cancer staging. Eur J Cardiothorac Surg. 2009;35:332-5.

11. Groth SS, Andrade RS. Endobronchial and endoscopic ultrasound-guided fine needle aspiration: a must for thoracic surgeons. Ann Thorac Surg. 2010;89: S2079-83.

12. McLaren DG, Novakofski J, Parrett DF, Lo LL, Singh SD, Neumann KR, et al. A study of operator effects on ultrasonic measures of fat depth and longissimus muscle area in cattle, sheep and pigs. J Anim Sci. 1991;69:54-66. 